\title{
Dihydropyrimidine Dehydrogenase Deficiency
}

National Cancer Institute

\section{Source}

National Cancer Institute. Dihydropyrimidine Dehydrogenase Deficiency. NCI Thesaurus.

Code C84672.

A rare disorder characterized by an inborn error in pyrimidine metabolism. It results in the deficiency or complete absence of the enzyme dihydropyrimidine dehydrogenase.

Individuals with this disorder may develop a severe toxicity reaction if they are exposed to the chemotherapeutic agent 5-fluorouracil. 